\title{
Construction of nonuniform periodic wavelet frames on non-Archimedean fields
}

\begin{abstract}
In real life applications not all signals are obtained by uniform shifts; so there is a natural question regarding analysis and decompositions of these types of signals by a stable mathematical tool. Gabardo and Nashed, and Gabardo and $\mathrm{Yu}$ filled this gap by the concept of nonuniform multiresolution analysis and nonuniform wavelets based on the theory of spectral pairs for which the associated translation set $\Lambda=\{0, r / N\}+2 \mathbb{Z}$ is no longer a discrete subgroup of $\mathbb{R}$ but a spectrum associated with a certain one-dimensional spectral pair and the associated dilation is an even positive integer related to the given spectral pair. In this paper, we introduce a notion of nonuniform periodic wavelet frame on non-Archimedean field. Using the Fourier transform technique and the unitary extension principle, we propose an approach for the construction of nonuniform periodic wavelet frames on non-Archimedean fields.
\end{abstract}

1. Introduction. The notion of frames was first introduced by Duffin and Shaeffer [10] in connection with some deep problems in nonharmonic Fourier series and more particularly, with the question of determining when a family of exponentials $\left\{e^{i \alpha_{n} t}: n \in \mathbb{Z}\right\}$ is complete for $L^{2}[a, b]$. Frames did not generate much interest outside nonharmonic Fourier series until the seminal work by Daubechies et al. [9]. After their pioneer work, the theory of frames began to be studied widely and deeply, particularly in the more specialized context of wavelet frames and Gabor frames. Frames provide a useful model

2010 Mathematics Subject Classification. 42C40, 42C15, 43A70, 11S85.

Key words and phrases. Nonuniform frame, wavelet mask, scaling function, Fourier transform. 
to obtain signal decompositions in cases where redundancy, robustness, oversampling and irregular sampling occur.

Today, the theory of frames has become an interesting and fruitful field of mathematics with abundant applications in signal processing, image processing, harmonic analysis, Banach space theory, sampling theory, wireless sensor networks, optics, filter banks, quantum computing, and medicine. An important example of frames is a wavelet frame, which is obtained by translating and dilating a finite family of functions. One of the most useful methods to construct wavelet frames is through the concept of unitary extension principle (UEP) introduced by Ron and Shen [17] and subsequently extended by Daubechies et al. [8] in the form of the Oblique Extension Principle (OEP). They gave sufficient conditions for constructing tight and dual wavelet frames for any given refinable function $\phi(x)$ which generates a multiresolution analysis. Gabardo and Nashed [11], and Gabardo and Yu [12] introduced the notion of nonuniform multiresolution analysis and nonuniform wavelets based on the theory of spectral pairs for which the associated translation set $\Lambda=\{0, r / N\}+2 \mathbb{Z}$ is no longer a discrete subgroup of $\mathbb{R}$ but a spectrum associated with a certain one-dimensional spectral pair and the associated dilation is an even positive integer related to the given spectral pair.

In recent years, there has been a considerable interest in the problem of constructing periodic wavelet bases and frames in Hilbert spaces as most of the signals of practical interest are periodic in nature. Apart from signals that are inherently periodic, all signals resulting from experiments with a finite duration can in principle be modeled as periodic signals [14]. The setup of tight wavelet frames provides great flexibility in approximating and representing periodic functions. Using periodization techniques, Zhang [25] constructed a dual pair of periodic wavelet frames for $L^{2}[0,1]$ under the assumption that the support of the wavelet function $\psi$ in the frequency domain is contained in $[-\pi,-\varepsilon] \cup[\varepsilon, \pi], \varepsilon>0$. Zhang and Saito [26] have constructed general periodic wavelet frames for $L^{2}[0,1]$ using extension principles. Later on, $\mathrm{Lu}$ and $\mathrm{Li}$ [15] constructed periodic wavelet frames with dilation matrix.

On the other hand, the past decade has also witnessed a tremendous interest in the problem of constructing wavelet bases and frames on various spaces other than $\mathbb{R}$. For example, R. L. Benedetto and J. J. Benedetto [6] developed a wavelet theory for local fields and related groups. They did not develop the multiresolution analysis (MRA) approach, their method is based on the theory of wavelet sets and only allows the construction of wavelet functions whose Fourier transforms are characteristic functions of some sets. Jiang et al. [13] pointed out a method for constructing orthogonal wavelets on a local field $\mathbb{K}$ with a constant generating sequence and derived necessary and sufficient conditions for a solution of the refinement equation 
to generate a multiresolution analysis of $L^{2}(\mathbb{K})$. Subsequently, tight wavelet frames on local fields of the positive characteristic were constructed by Shah and Debnath [23] using extension principles. In the series of papers $[1,2,3$, $4,5,19,20,21,22]$, we have obtained various results related to wavelet and Gabor frames on non-Archimedean local fields.

Drawing inspiration from the above work, our aim is to extend the notion of wavelet frames to nonuniform periodic wavelet frames on non-Archimedean fields via extension principles. More precisely, we prove that under some mild conditions, the periodization of any nonuniform wavelet frame constructed by the unitary extension principle is a nonuniform periodic wavelet frame on non-Archimedean fields.

The layout of this paper is as follows. In Section 2, we discuss some preliminary facts about non-Archimedean fields and also some results which are required in the subsequent sections. Sections 3 is devoted to our main results about nonuniform periodic wavelet frames.

\section{Preliminaries and nonuniform periodic wavelet system on non-} Archimedean fields. A non-Archimedean field $\mathbb{K}$ is a locally compact, nondiscrete and totally disconnected field. If it is of the characteristic zero, then it is a field of $p$-adic numbers $\mathbb{Q}_{p}$ or its finite extension. If $\mathbb{K}$ is of the positive characteristic, then $\mathbb{K}$ is a field of formal Laurent series over a finite field $G F\left(p^{c}\right)$. If $c=1$, it is a $p$-series field, while for $c \neq 1$, it is an algebraic extension of degree $c$ of a $p$-series field. Let $\mathbb{K}$ be a fixed non-Archimedean field with the ring of integers $\mathfrak{D}=\{x \in \mathbb{K}:|x| \leq 1\}$. Since $\mathbb{K}^{+}$is a locally compact Abelian group, we choose a Haar measure $d x$ for $\mathbb{K}^{+}$. The field $\mathbb{K}$ is a locally compact, nontrivial, totally disconnected and complete topological field endowed with non-Archimedean norm $|\cdot|: \mathbb{K} \rightarrow \mathbb{R}^{+}$satisfying

(a) $|x|=0$ if and only if $x=0$;

(b) $|x y|=|x||y|$ for all $x, y \in \mathbb{K}$;

(c) $|x+y| \leq \max \{|x|,|y|\}$ for all $x, y \in \mathbb{K}$.

Property (c) is called the ultrametric inequality. Let $\mathfrak{B}=\{x \in \mathbb{K}:|x|<1\}$ be the prime ideal of the ring of integers $\mathfrak{D}$ in $\mathbb{K}$. Then, the residue space $\mathfrak{D} / \mathfrak{B}$ is isomorphic to a finite field $G F(q)$, where $q=p^{c}$ for some prime $p$ and $c \in \mathbb{N}$. Since $\mathbb{K}$ is totally disconnected and $\mathfrak{B}$ is both prime and principal ideal, there exists a prime element $\mathfrak{p}$ of $\mathbb{K}$ such that $\mathfrak{B}=\langle\mathfrak{p}\rangle=\mathfrak{p} \mathfrak{D}$. Let $\mathfrak{D}^{*}=\mathfrak{D} \backslash \mathfrak{B}=\{x \in \mathbb{K}:|x|=1\}$. Clearly, $\mathfrak{D}^{*}$ is a group of units in $\mathbb{K}^{*}$ and if $x \neq 0$, then we can write $x=\mathfrak{p}^{n} y, y \in \mathfrak{D}^{*}$. Moreover, if $\mathcal{U}=\left\{a_{m}: m=0,1, \ldots, q-1\right\}$ denotes the fixed full set of coset representatives of $\mathfrak{B}$ in $\mathfrak{D}$, then every element $x \in \mathbb{K}$ can be expressed uniquely as $x=\sum_{\ell=k}^{\infty} c_{\ell} \mathfrak{p}^{\ell}$ with $c_{\ell} \in \mathcal{U}$. Recall that $\mathfrak{B}$ is compact and open, so each fractional ideal $\mathfrak{B}^{k}=\mathfrak{p}^{k} \mathfrak{D}=\left\{x \in \mathbb{K}:|x|<q^{-k}\right\}$ is also compact and open 
and is a subgroup of $\mathbb{K}^{+}$. We use the notation from Taibleson's book [13]. In the rest of this paper, we use the symbols $\mathbb{N}, \mathbb{N}_{0}$ and $\mathbb{Z}$ to denote the sets of natural, nonnegative integers and integers, respectively.

Let $\chi$ be a fixed character on $\mathbb{K}^{+}$that is trivial on $\mathfrak{D}$ but nontrivial on $\mathfrak{B}^{-1}$. Therefore, $\chi$ is constant on cosets of $\mathfrak{D}$, so if $y \in \mathfrak{B}^{k}$, then $\chi_{y}(x)=\chi(y, x), x \in \mathbb{K}$. Suppose that $\chi_{u}$ is any character on $\mathbb{K}^{+}$, then the restriction $\chi_{u} \mid \mathfrak{D}$ is a character on $\mathfrak{D}$. Moreover, as characters on $\mathfrak{D}$, $\chi_{u}=\chi_{v}$ if and only if $u-v \in \mathfrak{D}$. Hence, if $\left\{u(n): n \in \mathbb{N}_{0}\right\}$ is a complete list of distinct coset representative of $\mathfrak{D}$ in $\mathbb{K}^{+}$, then, as it was proved in [13], the set $\left\{\chi_{u(n)}: n \in \mathbb{N}_{0}\right\}$ of distinct characters on $\mathfrak{D}$ is a complete orthonormal system on $\mathfrak{D}$.

We now impose a natural order on the sequence $\{u(n)\}_{n=0}^{\infty}$. We have $\mathfrak{D} / \mathfrak{B} \cong G F(q)$ where $G F(q)$ is a $c$-dimensional vector space over the field $G F(p)$. We choose a set $\left\{1=\zeta_{0}, \zeta_{1}, \zeta_{2}, \ldots, \zeta_{c-1}\right\} \subset \mathfrak{D}^{*}$ such that $\operatorname{span}\left\{\zeta_{j}\right\}_{j=0}^{c-1} \cong G F(q)$. For $n \in \mathbb{N}_{0}$ satisfying

$0 \leq n<q, n=a_{0}+a_{1} p+\cdots+a_{c-1} p^{c-1}, 0 \leq a_{k}<p$, and $k=0,1, \ldots, c-1$,

we define

$$
u(n)=\left(a_{0}+a_{1} \zeta_{1}+\cdots+a_{c-1} \zeta_{c-1}\right) \mathfrak{p}^{-1} .
$$

Also, for $n=b_{0}+b_{1} q+b_{2} q^{2}+\cdots+b_{s} q^{s}, n \in \mathbb{N}_{0}, 0 \leq b_{k}<q, k=$ $0,1,2, \ldots, s$, we set

$$
u(n)=u\left(b_{0}\right)+u\left(b_{1}\right) \mathfrak{p}^{-1}+\cdots+u\left(b_{s}\right) \mathfrak{p}^{-s} .
$$

This defines $u(n)$ for all $n \in \mathbb{N}_{0}$. In general, it is not true that $u(m+n)=$ $u(m)+u(n)$. But, if $r, k \in \mathbb{N}_{0}$ and $0 \leq s<q^{k}$, then $u\left(r q^{k}+s\right)=u(r) \mathfrak{p}^{-k}+$ $u(s)$. Further, it is also easy to verify that $u(n)=0$ if and only if $n=0$ and $\left\{u(\ell)+u(k): k \in \mathbb{N}_{0}\right\}=\left\{u(k): k \in \mathbb{N}_{0}\right\}$ for a fixed $\ell \in \mathbb{N}_{0}$. Hereafter we use the notation $\chi_{n}=\chi_{u(n)}, n \geq 0$.

Let the local field $\mathbb{K}$ be of the characteristic $p>0$ and $\zeta_{0}, \zeta_{1}, \zeta_{2}, \ldots, \zeta_{c-1}$ be as above. We define a character $\chi$ on $\mathbb{K}$ as follows:

$$
\chi\left(\zeta_{\mu} \mathfrak{p}^{-j}\right)= \begin{cases}\exp (2 \pi i / p), & \mu=0 \text { and } j=1 \\ 1, & \mu=1, \ldots, c-1 \text { or } j \neq 1 .\end{cases}
$$

The Fourier transform of $f \in L^{1}(\mathbb{K})$ is denoted by $\hat{f}(\xi)$ and defined by

$$
\mathcal{F}\{f(x)\}=\hat{f}(\xi)=\int_{\mathbb{K}} f(x) \overline{\chi_{\xi}(x)} d x .
$$

It is noted that

$$
\hat{f}(\xi)=\int_{\mathbb{K}} f(x) \overline{\chi_{\xi}(x)} d x=\int_{\mathbb{K}} f(x) \chi(-\xi x) d x .
$$


The properties of Fourier transforms on non-Archimedean field $\mathbb{K}$ are much similar to those of on the classical field $\mathbb{R}$. In fact, the Fourier transform on non-Archimedean fields of the positive characteristic have the following properties:

- The map $f \mapsto \hat{f}$ is a bounded linear transformation of $L^{1}(\mathbb{K})$ into $L^{\infty}(\mathbb{K})$ and $\|\hat{f}\|_{\infty} \leq\|f\|_{1}$.

- If $f \in L^{1}(\mathbb{K})$, then $\hat{f}$ is uniformly continuous.

- If $f \in L^{1}(\mathbb{K}) \cap L^{2}(\mathbb{K})$, then $\|\hat{f}\|_{2}=\|f\|_{2}$.

The Fourier transform of a function $f \in L^{2}(\mathbb{K})$ is defined by

$$
\hat{f}(\xi)=\lim _{k \rightarrow \infty} \hat{f}_{k}(\xi)=\lim _{k \rightarrow \infty} \int_{|x| \leq q^{k}} f(x) \overline{\chi_{\xi}(x)} d x,
$$

where $f_{k}=f \Phi_{-k}$ and $\Phi_{k}$ is the characteristic function of $\mathfrak{B}^{k}$. Furthermore, if $f \in L^{2}(\mathfrak{D})$, then we define the Fourier coefficients of $f$ as

$$
\hat{f}(u(n))=\int_{\mathfrak{D}} f(x) \overline{\chi_{u(n)}(x)} d x .
$$

The series $\sum_{n \in \mathbb{N}_{0}} \hat{f}(u(n)) \chi_{u(n)}(x)$ is called the Fourier series of $f$. From the standard $L^{2}$-theory for compact Abelian groups, we conclude that the Fourier series of $f$ converges to $f$ in $L^{2}(\mathfrak{D})$ and Parseval's identity holds:

$$
\|f\|_{2}^{2}=\int_{\mathfrak{D}}|f(x)|^{2} d x=\sum_{n \in \mathbb{N}_{0}}|\hat{f}(u(n))|^{2} .
$$

We also denote the test function space on $\mathbb{K}$ by $\Omega(\mathbb{K})$, that is, each function $f$ in $\Omega(\mathbb{K})$ is a finite linear combination of functions of the form $\mathbf{1}_{k}(x-h), h \in \mathbb{K}, k \in \mathbb{Z}$, where $\mathbf{1}_{k}$ is the characteristic function of $\mathfrak{B}^{k}$. This class of functions can also be described in the following way. A function $g \in \Omega(\mathbb{K})$ if and only if there exist integers $k, \ell$ such that $g$ is constant on cosets of $\mathfrak{B}^{k}$ and is supported on $\mathfrak{B}^{\ell}$. It follows that $\Omega$ is closed under the Fourier transform and is an algebra of continuous functions with compact supports, which is dense in $\mathcal{C}_{0}(\mathbb{K})$ as well as in $L^{p}(\mathbb{K}), 1 \leq p<\infty$. For more details we refer to $[16,24]$.

For an integer $N \geq 1$ and an odd integer $r$ with $1 \leq r \leq q N-1$ such that $r$ and $N$ are relatively prime, we define

$$
\Lambda=\left\{0, \frac{u(r)}{N}\right\}+\mathcal{Z} .
$$

where $\mathcal{Z}=\left\{u(n): n \in \mathbb{N}_{0}\right\}$. It is easy to verify that $\Lambda$ is not a group on non-Archimedean field $\mathbb{K}$, but is the union of $\mathcal{Z}$ and a translate of $\mathcal{Z}$.

As in the standard scheme, one expects existence of $q N-1$ functions so that their translation by elements of $\Lambda$ and dilations by the integral powers of $\mathfrak{p}^{-1} N$ form an orthonormal basis for $L^{2}(\mathbb{K})$. 
For $j \in \mathbb{N}_{0}$ let $\mathcal{N}_{j}$ denote a full collection of coset representatives of $\Lambda /(q N)^{j} \Lambda$, i.e.

$$
\mathcal{N}_{j}=\left\{0,1,2, \ldots,(q N)^{j}-1\right\}, \quad j \geq 0 .
$$

Then, $\Lambda=\bigcup_{n \in \mathcal{N}_{j}}\left(n+(q N)^{j} \Lambda\right)$ and for any distinct $n_{1}, n_{2} \in \mathcal{N}_{j}$, we have $\left(n_{1}+(q N)^{j} \Lambda\right) \cap\left(n_{2}+(q N)^{j} \Lambda\right)=\emptyset$. Thus, every nonnegative integer $k$ can uniquely be written as $k=r(q N)^{j}+s$, where $r \in \Lambda, s \in \mathcal{N}_{j}$. Further, a bounded function $W: \mathbb{K} \rightarrow \mathbb{K}$ is said to be a radial decreasing $L^{1}$-majorant of $f(x) \in L^{2}(\mathbb{K})$ if $|f(x)| \leq W(x), W \in L^{1}(\mathbb{K})$ and $W(0)<\infty$.

Let $a$ and $b$ be any two fixed elements in $\mathbb{K}$. Then, for any prime $\mathfrak{p}$ and $m, n \in \mathbb{N}_{0}$, let $D_{\mathfrak{p}}, T_{u(n) a}$ and $E_{u(m) b}$ be the unitary operators acting on $f \in L^{2}(\mathbb{K})$ defined by:

$$
\begin{aligned}
T_{u(n) a} f(x) & =f(x-u(n) a) & & \text { (translation), } \\
E_{u(m) b} f(x) & =\chi(u(m) b x) f(x) & & \text { (modulation), } \\
D_{\mathfrak{p}} f(x) & =\sqrt{q N} f\left(\mathfrak{p}^{-1} N x\right) & & \text { (dilation). }
\end{aligned}
$$

For given $\Psi:=\left\{\psi_{1}, \ldots, \psi_{q N-1}\right\} \subset L^{2}(\mathbb{K})$, define the nonuniform wavelet system:

$$
\begin{aligned}
& \mathcal{W}(\Psi, \lambda) \\
& =\left\{\psi_{\ell, j, \lambda}:=(q N)^{j / 2} \psi_{\ell}\left(\left(\mathfrak{p}^{-1} N\right)^{j} x-\lambda\right), j \in \mathbb{Z}, \lambda \in \Lambda, 1 \leq \ell \leq q N-1\right\} .
\end{aligned}
$$

The nonuniform wavelet system $\mathcal{W}(\Psi, \lambda)$ is called a nonuniform wavelet frame, if there exist positive numbers $0<A \leq B<\infty$ such that for all $f \in L^{2}(\mathbb{K})$

$$
A\|f\|_{2}^{2} \leq \sum_{\ell=1}^{q N-1} \sum_{j \in \mathbb{Z}} \sum_{\lambda \in \Lambda}\left|\left\langle f, \psi_{\ell, j, \lambda}\right\rangle\right|^{2} \leq B\|f\|_{2}^{2} .
$$

The largest $A$ and the smallest $B$ for which (2.8) holds are called nonuniform wavelet frame bounds. A wavelet frame is a tight non uniform wavelet frame if $A$ and $B$ are chosen such that $A=B$ and then the generators $\left\{\psi_{1}, \psi_{2}, \ldots, \psi_{q N-1}\right\}$ are often referred to as tight nonuniform framelets. If only the right-hand inequality in $(2.8)$ holds, then $\mathcal{W}(\Psi, \lambda)$ is called a Bessel sequence.

Next, we give a brief account of the MRA-based wavelet frames generated by the wavelet masks on non-Archimedean local fields. Following the unitary extension principle, one often starts with a refinable function or even with a refinement mask to construct desired wavelet frames. A function $\varphi \in$ $L^{2}(\mathbb{K})$ is called a nonuniform refinable function, if it satisfies an equation of the type

$$
\varphi(x)=\sqrt{q N} \sum_{\lambda \in \Lambda} a_{\lambda} \varphi\left(\left(\mathfrak{p}^{-1} N\right) x-\lambda\right)
$$


where $\left\{a_{\lambda}: \lambda \in \Lambda\right\} \in l^{2}\left(\mathbb{N}_{0}\right)$. In the frequency domain, equation (2.9) can be written as

$$
\hat{\varphi}(\xi)=m_{0}\left(\frac{\mathfrak{p} \xi}{N}\right) \hat{\varphi}\left(\frac{\mathfrak{p} \xi}{N}\right)
$$

where

$$
m_{0}(\xi)=\frac{1}{\sqrt{q N}} \sum_{\lambda \in \Lambda} a_{\lambda} \overline{\chi_{\lambda}(\xi)}
$$

is an integral periodic function in $L^{2}(\mathfrak{D})$ and is often called the refinement mask of $\varphi$. Observe that $\chi_{k}(0)=\hat{\phi}(0)=1$. By letting $\xi=0$ in equations (2.10) and (2.11), we obtain $\sum_{\lambda \in \Lambda} a_{\lambda}=1$. Further, it is proved in [23] that a function $\varphi \in L^{2}(\mathbb{K})$ generates an MRA in $L^{2}(\mathbb{K})$ if and only if

$$
\sum_{\lambda \in \Lambda}|\hat{\varphi}(\xi+\lambda)|^{2}=1, \text { for a.e. } \xi \in \mathfrak{D} \text { and } \hat{\varphi}(0)=\lim _{\xi \rightarrow 0} \hat{\varphi}(\xi)=1, \xi \in \mathbb{K} \text {. }
$$

Suppose $\Psi=\left\{\psi_{1}, \ldots, \psi_{q N-1}\right\}$ is a set of MRA functions derived from

$$
\hat{\psi}_{\ell}(\xi)=m_{\ell}\left(\frac{\mathfrak{p} \xi}{N}\right) \hat{\varphi}\left(\frac{\mathfrak{p} \xi}{N}\right)
$$

where

$$
m_{\ell}(\xi)=\frac{1}{\sqrt{q N}} \sum_{\lambda \in \Lambda} a_{\lambda}^{\ell} \overline{\chi_{\lambda}(\xi)}, \quad 1 \leq \ell \leq q N-1
$$

are the integral periodic functions in $L^{2}(\mathfrak{D})$ and are called the nonuniform framelet symbols or nonuniform wavelet masks. With $m_{\ell}(\xi), 0 \leq \ell \leq q N-1$ as the wavelet masks, we formulate the matrix $\mathcal{M}(\xi)$ as

$$
\mathcal{M}(\xi)=\left(\begin{array}{cccc}
m_{0}(\xi) & m_{0}(\xi+\mathfrak{p} u(1)) & \ldots & m_{0}(\xi+\mathfrak{p} u(s-1)) \\
m_{1}(\xi) & m_{1}(\xi+\mathfrak{p} u(1)) & \ldots & m_{1}(\xi+\mathfrak{p} u(s-1)) \\
\vdots & \vdots & \ddots & \vdots \\
m_{q N-1}(\xi) & m_{q N-1}(\xi+\mathfrak{p} u(1)) & \ldots & m_{q N-1}(\xi+\mathfrak{p} u(s-1))
\end{array}\right)
$$

The so-called unitary extension principle (UEP) provides a sufficient condition on $\Psi=\left\{\psi_{1}, \psi_{2} \ldots, \psi_{q N-1}\right\}$ such that the nonuniform wavelet system $\mathcal{W}(\Psi, \lambda)$ given by $(2.7)$ constitutes a tight frame for $L^{2}(\mathbb{K})$. It is well known that in order to apply the UEP to derive wavelet tight frame from a given refinable function, the corresponding refinement mask must satisfy

$$
\sum_{\lambda=0}^{q N-1}\left|m_{0}(\xi+q N \lambda)\right|^{2} \leq 1, \quad \xi \in \mathbb{K} .
$$

In this connection, Shah and Debnath [23] gave an explicit construction scheme for the construction of tight wavelet frames on local fields of the positive characteristic using unitary extension principles. The following is 
the fundamental tool they gave to construct tight wavelet frames on local fields.

Theorem 2.1. Let $\varphi(x)$ be a compactly supported refinable function and $\hat{\phi}(0)=1$. Then, the nonuniform wavelet system $\mathcal{W}(\Psi, \lambda)$ given by (2.7) constitutes a normalized tight wavelet frame in $L^{2}(\mathbb{K})$ provided the matrix $\mathcal{M}(\xi)$ defined in (2.15) satisfies

$$
\mathcal{M}(\xi) \mathcal{M}^{*}(\xi)=I_{q} \quad \text { for a.e. } \xi \in \sigma\left(V_{0}\right)
$$

where $\sigma\left(V_{0}\right):=\left\{\xi \in \mathfrak{D}: \sum_{\lambda \in \Lambda}|\hat{\varphi}(\xi+\lambda)|^{2} \neq 0\right\}$.

3. Nonuniform periodic wavelet frames on non-Archimedean fields. In this section, we present an approach for constructing nonuniform periodic wavelet frames on non-Archimedean fields by virtue of the unitary extension principle (UEP).

For any $f \in L^{1}(\mathbb{K})$, we define the periodic version of $f$ as

$$
f^{\text {per }}=\sum_{\lambda \in \Lambda} f(x+\lambda u(N)) .
$$

It is easy to see that $f^{\text {per }}$ is well defined and it is an $\mathbb{N}$-periodic local integrable function. With same dilation and translation operators as defined in Section 2, we define the nonuniform periodic wavelet system as

$$
\mathcal{W}^{\text {per }}(\Psi, \lambda):=\left\{\varphi^{\text {per }}, \psi_{\ell, j, \lambda}^{\text {per }}: 1 \leq \ell \leq q N-1, j \in \mathbb{N}_{0}, \lambda \in \mathcal{N}_{j}\right\} .
$$

In order to establish the main result of this section, we first state and prove the following lemmas.

Lemma 3.1. Suppose that the nonuniform periodic wavelet system $\mathcal{W}^{\text {per }}(\Psi, \lambda)$ is defined by (3.1). Then, for any periodic function $f$ and given $\varepsilon>0$, there exists a positive integer $J \in \mathbb{N}$ such that

$$
(1-\varepsilon)\|f\|_{2}^{2} \leq \sum_{\lambda=0}^{(q N)^{j}-1}\left|\left\langle f, \varphi_{j, \lambda}^{p e r}\right\rangle\right|^{2} \leq(1+\varepsilon)\|f\|_{2}^{2} \quad \text { for all } j \geq J .
$$

Proof. Let $\Gamma$ denote the support of the Fourier coefficients $\left\{\hat{f}\left(u\left(\frac{r}{N}\right)\right)\right\}_{r \in \mathbb{N}_{0}}$. Then, we have

$$
f(x)=\sum_{r \in \Gamma} \hat{f}\left(u\left(\frac{r}{N}\right)\right) \overline{\chi_{r / N}(x)} .
$$

Assume that

$$
\varphi_{j, \lambda}^{\mathrm{per}}(x)=\sum_{r \in \mathbb{N}_{0}} \hat{\varphi}_{j, \lambda}^{\mathrm{per}}\left(u\left(\frac{r}{N}\right)\right) \overline{\chi_{r / N}(x)} .
$$

The Fourier coefficients in the above expression can be written as

$$
\hat{\varphi}_{j, \lambda}^{\text {per }}\left(u\left(\frac{r}{N}\right)\right)=(q N)^{j / 2} \hat{\varphi}\left(\left(\mathfrak{p}^{-1} N\right)^{-j} u\left(\frac{r}{N}\right)\right) \overline{\chi_{\lambda}\left(\left(\mathfrak{p}^{-1} N\right)^{-j} u\left(\frac{r}{N}\right)\right)} .
$$


Parseval's formula of the above Fourier series gives

$$
\begin{aligned}
& \sum_{\lambda=0}^{(q N)^{j}-1}\left|\left\langle f, \varphi_{j, \lambda}^{\mathrm{per}}\right\rangle\right|^{2}=\sum_{\lambda=0}^{(q N)^{j}-1}\left|\sum_{r \in \Gamma} \hat{f}(u(r)) \overline{\hat{\varphi}_{j, \lambda}^{\mathrm{per}}(u(r))}\right|^{2} \\
& =\sum_{\lambda=0}^{(q N)^{j}-1}\left|\sum_{r \in \Gamma} \hat{f}(u(r))(q N)^{j / 2} \overline{\hat{\varphi}\left(\left(\mathfrak{p}^{-1} N\right)^{-j} u(r)\right)} \chi_{\lambda}\left(\left(\mathfrak{p}^{-1} N\right)^{-j} u(r)\right)\right|^{2} \\
& =\sum_{\lambda=0}^{(q N)^{j}-1}\left|\sum_{r \in \Gamma} d_{r}(\hat{f}, \hat{\varphi}) \chi_{\lambda}\left(\left(\mathfrak{p}^{-1} N\right)^{-j} u(r)\right)\right|^{2}
\end{aligned}
$$

where $d_{r}(\hat{f}, \hat{\varphi})=(q N)^{j / 2} \hat{f}(u(r)) \overline{\hat{\varphi}\left(\left(\mathfrak{p}^{-1} N\right)^{-j} u(r)\right)}$. Since $\Gamma$ is a finite set, there exists a positive number $N$ such that $E \subseteq D(N):=\{\lambda \in \Lambda:|\lambda| \leq N\}$. Hence, there exists $J_{1} \geq 0$ such that for all $j \geq J_{1}$, the elements of $D(N)$ lie in different cosets of $\Lambda /(q N)^{j} \Lambda$. Thus, the cardinality of $\Gamma \cap\left(\lambda+(q N)^{j} \Lambda\right)$ is at most one for each $j \geq J_{1}, \lambda \in \mathcal{N}_{j}$. Consequently, we have

$$
\begin{aligned}
& \sum_{\lambda=0}^{(q N)^{j}-1}\left|\left\langle f, \varphi_{j, \lambda}^{\mathrm{per}}\right\rangle\right|^{2} \\
= & \sum_{\lambda=0}^{(q N)^{j}-1} \sum_{r \in \Gamma} d_{r}(\hat{f}, \hat{\varphi}) \chi_{\lambda}\left(\left(\mathfrak{p}^{-1} N\right)^{-j} u(r)\right) \sum_{s \in \Gamma} \overline{d_{s}(\hat{f}, \hat{\varphi}) \chi_{\lambda}\left(\left(\mathfrak{p}^{-1} N\right)^{-j} u(s)\right)} \\
= & \sum_{r \in \Gamma} \sum_{s \in \Gamma} d_{r}(\hat{f}, \hat{\varphi}) \overline{d_{r}(\hat{f}, \hat{\varphi})} \sum_{\lambda=0}^{(q N)^{j}-1} \chi_{\lambda}\left(\left(\mathfrak{p}^{-1} N\right)^{-j} u(r-s)\right) \\
= & (q N)^{j} \sum_{s \in \Gamma}\left|d_{s}(\hat{f}, \hat{\varphi})\right|^{2} \\
= & \sum_{s \in \Gamma}\left|(q N)^{j / 2} \hat{f}(u(s)) \overline{\hat{\varphi}\left(\left(\mathfrak{p}^{-1} N\right)^{-j} u(s)\right)}\right|^{2} .
\end{aligned}
$$

Since $\hat{\varphi}(0)=\lim _{\xi \rightarrow 0} \hat{\varphi}(\xi)=1$, therefore there exists a nonnegative integer $J_{2}$ such that

$$
(1-\varepsilon) \leq\left|\hat{\varphi}\left(\left(\mathfrak{p}^{-1} N\right)^{-j} u(s)\right)\right|^{2} \leq(1+\varepsilon) \quad \text { for all } j \geq J_{2} .
$$

Let $J=\max \left\{J_{1}, J_{2}\right\}$, then with this choice of $j \geq J$, we obtain

$$
(1-\varepsilon) \sum_{s \in \Gamma}|\hat{f}(u(s))|^{2} \leq \sum_{\lambda=0}^{(q N)^{j}-1}\left|\left\langle f, \varphi_{j, \lambda}^{\mathrm{per}}\right\rangle\right|^{2} \leq(1+\varepsilon) \sum_{s \in \Gamma}|\hat{f}(u(s))|^{2},
$$


which implies that

$$
(1-\varepsilon)\|f\|_{2}^{2} \leq \sum_{\lambda=0}^{(q N)^{j}-1}\left|\left\langle f, \varphi_{j, \lambda}^{\mathrm{per}}\right\rangle\right|^{2} \leq(1+\varepsilon)\|f\|_{2}^{2} .
$$

This completes the proof of Lemma 3.1.

Lemma 3.2. Let $\varphi$ defined by (2.11) be the nonuniform refinable function with $m_{0}(\xi)$ as its refinement mask and let $m_{\ell}(\xi), 1 \leq \ell \leq q N-1$, be the wavelet masks. The nonuniform wavelet system $\mathcal{W}(\Psi, \lambda)$ given by $(2.7)$ form a normalized tight frame for $L^{2}(\mathbb{K})$. Then, for any function $f \in L^{2}(\mathbb{K})$ we have

$$
\sum_{\lambda \in \Lambda}\left|\left\langle f, \varphi_{j+1, \lambda}\right\rangle\right|^{2}=\sum_{\lambda \in \Lambda}\left|\left\langle f, \varphi_{j, \lambda}\right\rangle\right|^{2}+\sum_{\ell=1}^{q N-1} \sum_{\lambda \in \Lambda}\left|\left\langle f, \psi_{\ell, j, \lambda}\right\rangle\right|^{2} .
$$

Proof. For any $f \in L^{2}(\mathbb{K})$ and $j \in \mathbb{N}_{0}$, define the linear operators $\mathcal{P}_{j}$ and $\mathcal{Q}_{j}$ as:

$$
\mathcal{P}_{j} f(x)=\sum_{\lambda \in \Lambda}\left\langle f, \varphi_{j, \lambda}\right\rangle \varphi_{j, \lambda}(x), \mathcal{Q}_{j} f(x)=\sum_{\ell=1}^{q N-1} \sum_{\lambda \in \Lambda}\left\langle f, \psi_{\ell, j, \lambda}\right\rangle \psi_{\ell, j, \lambda}(x) .
$$

Since $\Omega(\mathbb{K})$ is dense in $L^{2}(\mathbb{K})$ and closed under the Fourier transform, it is sufficient to prove that

$$
\left\langle\mathcal{P}_{j} f, f\right\rangle+\left\langle\mathcal{Q}_{j} f, f\right\rangle=\left\langle\mathcal{P}_{j+1} f, f\right\rangle
$$

holds for all the functions $f$ in $\Omega(\mathbb{K})$. Therefore, for all $f \in \Omega(\mathbb{K})$ and $j \in \mathbb{Z}$, $k \in \mathbb{N}_{0}$, using Parseval's identity, we obtain

$$
\begin{aligned}
& \left\langle\mathcal{P}_{j} f, f\right\rangle=(q N)^{j} \int_{\mathfrak{D}}\left|\sum_{r \in \mathbb{N}_{0}} \hat{f}\left(\left(\mathfrak{p}^{-1} N\right)^{-j}(\xi+u(r))\right) \overline{\hat{\varphi}(\xi+u(r))}\right|^{2} d \xi \\
& =\int_{(\mathfrak{p}-1 N)^{-j} \mathfrak{D}}\left|\sum_{r \in \mathbb{N}_{0}} \hat{f}\left(\xi+\left(\mathfrak{p}^{-1} N\right)^{-j} u(r)\right) \overline{\hat{\varphi}\left(\left(\mathfrak{p}^{-1} N\right)^{j} \xi+u(r)\right)}\right|^{2} d \xi .
\end{aligned}
$$

Since $m_{0}(\xi)$ is an integral-periodic function, equation (3.8) yields

$$
\begin{aligned}
&\left\langle\mathcal{P}_{j} f, f\right\rangle \\
&=\int_{\left(\mathfrak{p}^{-1} N\right)^{-j} \mathfrak{D}} \mid \sum_{r \in \mathbb{N}_{0}} \hat{f}\left(\xi+\left(\mathfrak{p}^{-1} N\right)^{-j} u(r)\right) \overline{\hat{\varphi}\left(\left(\mathfrak{p}^{-1} N\right)^{j+1} \xi+\left(\mathfrak{p}^{-1} N\right) u(r)\right)} \\
& \times\left.\overline{m_{0}\left(\left(\mathfrak{p}^{-1} N\right)^{j+1} \xi+\left(\mathfrak{p}^{-1} N\right) u(r)\right)}\right|^{2} d \xi
\end{aligned}
$$




$$
\begin{aligned}
& =\int_{\left(\mathfrak{p}^{-1} N\right)^{-j} \mathfrak{D}} \mid \sum_{r \in \mathbb{N}_{0}} \sum_{s=0}^{q N-1} \hat{f}\left(\xi+\left(\mathfrak{p}^{-1} N\right)^{-j}\left(\left(\mathfrak{p}^{-1} N\right) u(r)+u(s)\right)\right) \\
& \times \overline{\hat{\varphi}\left(\left(\mathfrak{p}^{-1} N\right)^{j+1} \xi+\left(\mathfrak{p}^{-1} N\right)\left(\left(\mathfrak{p}^{-1} N\right) u(r)+u(s)\right)\right)} \\
& \times\left.\overline{m_{0}\left(\left(\mathfrak{p}^{-1} N\right)^{j+1} \xi+\left(\mathfrak{p}^{-1} N\right)\left(\left(\mathfrak{p}^{-1} N\right) u(r)+u(s)\right)\right)}\right|^{2} d \xi \\
& =\int_{\left(\mathfrak{p}^{-1} N\right)^{-j} \mathfrak{D}} \mid \sum_{r \in \mathbb{N}_{0}} \sum_{s=0}^{q N-1} \hat{f}\left(\xi+\left(\mathfrak{p}^{-1} N\right)^{-j}\left(\left(\mathfrak{p}^{-1} N\right) u(r)+u(s)\right)\right) \\
& \times \overline{\hat{\varphi}\left(\left(\mathfrak{p}^{-1} N\right)^{j+1} \xi+\left(\mathfrak{p}^{-1} N\right)\left(\left(\mathfrak{p}^{-1} N\right) u(r)+u(s)\right)\right)} \\
& \times\left.\overline{m_{0}\left(\left(\mathfrak{p}^{-1} N\right)^{j+1} \xi+\left(\mathfrak{p}^{-1} N\right) u(s)\right)}\right|^{2} d \xi \\
& =\int_{(\mathfrak{p}-1 N)^{-j \mathfrak{D}}}\left|\sum_{s=0}^{q N-1} \mathcal{R}_{f, \varphi}^{j}(u(s), \xi) \overline{m_{0}\left(\mathfrak{p}^{j+1} \xi+\mathfrak{p} u(s)\right)}\right|^{2} d \xi,
\end{aligned}
$$

where

$$
\begin{aligned}
\mathcal{R}_{f, \varphi}^{j}(u(s), \xi)=\sum_{r \in \mathbb{N}_{0}} \hat{f} & \left(\xi+\left(\mathfrak{p}^{-1} N\right)^{-j}\left(\left(\mathfrak{p}^{-1} N\right) u(r)+u(s)\right)\right) \\
& \times \overline{\hat{\varphi}\left(\left(\mathfrak{p}^{-1} N\right)^{j+1} \xi+\left(\mathfrak{p}^{-1} N\right)\left(\left(\mathfrak{p}^{-1} N\right) u(r)+u(s)\right)\right)} .
\end{aligned}
$$

Proceeding in the similar manner as above, we can obtain

$$
\begin{aligned}
& \left\langle\mathcal{Q}_{j} f, f\right\rangle \\
& =\sum_{\ell=1}^{q N-1} \int_{\left(\mathfrak{p}^{-1} N\right)^{-j} \mathfrak{D}}\left|\sum_{s=0}^{q N-1} \mathcal{R}_{f, \varphi}^{j}(u(s), \xi) \overline{m_{\ell}\left(\left(\mathfrak{p}^{-1} N\right)^{j+1} \xi+\left(\mathfrak{p}^{-1} N\right) u(s)\right)}\right|^{2} d \xi
\end{aligned}
$$

Therefore, we have

$$
\begin{aligned}
&\left\langle\mathcal{P}_{j} f, f\right\rangle+\left\langle\mathcal{Q}_{j} f, f\right\rangle \\
&=\int_{\left(\mathfrak{p}^{-1} N\right)^{-j}}\left\{\frac{\left\{\sum_{s=0}^{q N-1} \mathcal{R}_{f, \varphi}^{j}(u(s), \xi) \overline{m_{0}\left(\left(\mathfrak{p}^{-1} N\right)^{j+1} \xi+\left(\mathfrak{p}^{-1} N\right) u(s)\right)}\right\}}{\left(\sum_{s^{\prime}=0}^{q N-1} \mathcal{R}_{f, \varphi}^{j}\left(u\left(s^{\prime}\right), \xi\right) \overline{m_{0}\left(\left(\mathfrak{p}^{-1} N\right)^{j+1} \xi+\left(\mathfrak{p}^{-1} N\right) u\left(s^{\prime}\right)\right)}\right\}} d \xi\right.
\end{aligned}
$$




$$
\begin{aligned}
& +\sum_{\ell=1}^{q N-1} \int_{\left(\mathfrak{p}^{-1} N\right)^{-j} \mathfrak{D}}\left\{\sum_{s=0}^{q N-1} \mathcal{R}_{f, \varphi}^{j}(u(s), \xi) \overline{m_{\ell}\left(\left(\mathfrak{p}^{-1} N\right)^{j+1} \xi+\left(\mathfrak{p}^{-1} N\right) u(s)\right)}\right\} \\
& \times \overline{\left\{\sum_{s^{\prime}=0}^{q N-1} \mathcal{R}_{f, \varphi}^{j}\left(u\left(s^{\prime}\right), \xi\right) \overline{m_{\ell}\left(\left(\mathfrak{p}^{-1} N\right)^{j+1} \xi+\left(\mathfrak{p}^{-1} N\right) u\left(s^{\prime}\right)\right)}\right\}} d \xi \\
& =\int_{\left(\mathfrak{p}^{-1} N\right)^{-j} \mathfrak{D}}\left\{\sum_{s=0}^{q N-1} \sum_{s^{\prime}=0}^{q N-1} \mathcal{R}_{f, \varphi}^{j}(u(s), \xi) \overline{\mathcal{R}_{f, \varphi}^{j}\left(u\left(s^{\prime}\right), \xi\right)}\right\} \\
& \times\left\{\sum_{\ell=0}^{q N-1} m_{\ell}\left(\left(\mathfrak{p}^{-1} N\right)^{j+1} \xi+\left(\mathfrak{p}^{-1} N\right) u\left(s^{\prime}\right)\right) \overline{m_{\ell}\left(\left(\mathfrak{p}^{-1} N\right)^{j+1} \xi+\left(\mathfrak{p}^{-1} N\right) u(s)\right)}\right\} d \xi .
\end{aligned}
$$

The unitary extension principle condition (2.17) is equivalent to

$$
\sum_{\ell=0}^{q N-1} m_{\ell}\left(\left(\mathfrak{p}^{-1} N\right)^{j+1} \xi+\left(\mathfrak{p}^{-1} N\right) u\left(s^{\prime}\right)\right) \overline{m_{\ell}\left(\left(\mathfrak{p}^{-1} N\right)^{j+1} \xi+\left(\mathfrak{p}^{-1} N\right) u(s)\right)}=\delta_{s, s^{\prime}} .
$$

Therefore, we have

$$
\begin{aligned}
& \left\langle\mathcal{P}_{j} f, f\right\rangle+\left\langle\mathcal{Q}_{j} f, f\right\rangle \\
& =\int_{\left(\mathfrak{p}^{-1}\right)^{-j} \mathfrak{D}} \sum_{s=0}^{q N-1}\left|\mathcal{R}_{f, \varphi}^{j}(u(s), \xi)\right|^{2} d \xi \\
& =\int_{\left(\mathfrak{p}^{-1}\right)^{-j} \mathfrak{D}} \sum_{s=0}^{q N-1} \mid \sum_{r \in \mathbb{N}_{0}} \hat{f}\left(\xi+\left(\mathfrak{p}^{-1} N\right)^{-j}\left(\left(\mathfrak{p}^{-1} N\right) u(r)+u(s)\right)\right) \\
& \quad \times\left.\overline{\hat{\varphi}\left(\left(\mathfrak{p}^{-1} N\right)^{j+1} \xi+\left(\mathfrak{p}^{-1} N\right)\left(\left(\mathfrak{p}^{-1} N\right) u(r)+u(s)\right)\right)}\right|^{2} d \xi \\
& =\sum_{s=0}^{q N-1} \int_{\left(\mathfrak{p}^{-1} N\right)^{-j} \mathfrak{D}+\left(\mathfrak{p}^{-1} N\right)^{-j} u(s)} \mid \sum_{r \in \mathbb{N}_{0}} \hat{f}\left(\xi+\left(\mathfrak{p}^{-1} N\right)^{-j-1} u(r)\right) \\
& \quad \times \frac{\hat{\varphi}\left(\left(\mathfrak{p}^{-1} N\right)^{j+1} \xi+u(r)\right)}{2} \mid d \xi \\
& =\int_{\left(\mathfrak{p}^{-1} N\right)^{-j-1} \mathfrak{D} \mid} \mid \sum_{r \in \mathbb{N}_{0}} \hat{f}\left(\xi+\left(\mathfrak{p}^{-1} N\right)^{-j-1} u(r)\right) \\
& =\left\langle\mathcal{P}_{j+1} f, f\right\rangle,
\end{aligned}
$$

and hence, we get the desired result (3.5). 
Lemma 3.3. Let $\varphi \in L^{2}(\mathbb{K})$ be a nonuniform refinable function with refinement mask $m_{0}(\xi)$ and let the wavelet system $\mathcal{W}(\Psi, \lambda)$ given by (2.7) constitutes a normalized tight frame for $L^{2}(\mathbb{K})$. If $\left\{\varphi, \psi_{1}, \ldots, \psi_{L}\right\} \subset L^{1}(\mathbb{K}) \cap$ $L^{2}(\mathbb{K})$ and $\varphi, \psi_{1}, \ldots, \psi_{q N-1}$ have a common radial decreasing $L^{1}$-majorant, then we have

$$
\begin{aligned}
\sum_{\lambda=0}^{(q N)^{j+1}-1} & \left|\left\langle f, \varphi_{j+1, \lambda}^{\text {per }}\right\rangle\right|^{2} \\
& =\sum_{\lambda=0}^{(q N)^{j}-1}\left|\left\langle f, \varphi_{j, \lambda}^{p e r}\right\rangle\right|^{2}+\sum_{\ell=1}^{q N-1} \sum_{\lambda=0}^{(q N)^{j}-1}\left|\left\langle f, \psi_{\ell, j, \lambda}^{p e r}\right\rangle\right|^{2} .
\end{aligned}
$$

Proof. For any $f \in \Omega(\mathbb{K})$ and $j \in \mathbb{N}_{0}$, we have

$$
\begin{aligned}
\sum_{\lambda=0}^{(q N)^{j}-1}\left|\left\langle f, \varphi_{j, \lambda}^{\mathrm{per}}\right\rangle\right|^{2} & =\sum_{\lambda=0}^{(q N)^{j}-1}\left|\left\langle f, \sum_{r \in \mathbb{N}_{0}} \varphi_{j, \lambda}(x+u(r))\right\rangle\right|^{2} \\
& =\sum_{\lambda=0}^{(q N) j-1}\left|\sum_{r \in \mathbb{N}_{0}}\left\langle f, \varphi_{j, \lambda}(x+u(r))\right\rangle\right|^{2}
\end{aligned}
$$

Furthermore, we have

$$
\begin{aligned}
\sum_{r \in \mathbb{N}_{0}} \int_{\mathfrak{D}}\left|f(x) \overline{\varphi_{j, \lambda}(x+u(r))}\right| d x & \leq\|f\|_{L^{\infty}(\mathfrak{D})} \int_{\mathbb{K}}\left|\varphi_{j, \lambda}(x)\right| d x \\
& =\|f\|_{L^{\infty}(\mathfrak{D})}(q N)^{j / 2} \int_{\mathbb{K}}|\varphi(x)| d x<\infty .
\end{aligned}
$$

This implies that the series

$$
\sum_{\lambda=0}^{\left(q N^{j}-1\right.} \sum_{r \in \mathbb{N}_{0}} \sum_{s \in \mathbb{N}_{0}}\left\langle f, \varphi_{j, \lambda}(x+u(r))\right\rangle \overline{\left\langle f, \varphi_{j, \lambda}(x+u(s))\right\rangle}
$$

is absolutely convergent. Therefore, the series can be rearranged as follows:

$$
\begin{aligned}
\sum_{\lambda=0}^{(q N)^{j}-1} & \left|\left\langle f, \varphi_{j, \lambda}^{\mathrm{per}}\right\rangle\right|^{2} \\
& =\sum_{\lambda=0}^{(q N)^{j}-1} \sum_{r \in \mathbb{N}_{0}} \sum_{s \in \mathbb{N}_{0}}\left\langle f, \varphi_{j, \lambda}(x+u(r))\right\rangle \overline{\left\langle f, \varphi_{j, \lambda}(x+u(s))\right\rangle} \\
& =\sum_{\lambda=0}^{(q N)^{j}-1} \sum_{r \in \mathbb{N}_{0}} \sum_{s \in \mathbb{N}_{0}}\left\langle f, \varphi_{j, \lambda}(x+u(r))\right\rangle \overline{\left\langle f, \varphi_{j, \lambda}(x+u(r)+u(s))\right\rangle} .
\end{aligned}
$$

For $s \in \mathbb{N}_{0}$, we define

$$
F_{s}(x)=f(x) \mathbf{1}_{\mathfrak{D}+u(s)}(x),
$$


where $\mathbf{1}_{\mathfrak{D}+u(s)}(x)$ is the characteristic function of the set $\mathfrak{D}+u(s)$. Using the fact that $\varphi_{j, \lambda}(x+u(s))=\varphi_{j, \lambda-\left(\mathfrak{p}^{-1} N\right)^{-j} u(s)}(x)$, we have

$$
\begin{aligned}
& \sum_{\lambda=0}^{(q N)^{j}-1}\left|\left\langle f, \varphi_{j, \lambda}^{\mathrm{per}}\right\rangle\right|^{2} \\
& =\sum_{\lambda=0}^{(q N)^{j}-1} \sum_{r \in \mathbb{N}_{0}} \sum_{s \in \mathbb{N}_{0}}\left\{\int_{\mathfrak{D}} f(x) \overline{\varphi_{j, \lambda}(x+u(r))} d x\right\} \\
& \times \overline{\left\{\int_{\mathfrak{D}} f(x) \overline{\varphi_{j, \lambda}(x+u(r)+u(s))} d x\right\}} \\
& =\sum_{\lambda=0}^{(q N)^{j}-1} \sum_{r \in \mathbb{N}_{0}} \sum_{s \in \mathbb{N}_{0}}\left\{\int_{\mathcal{D}} f(x) \overline{\varphi_{j, \lambda}(x+u(r))} d x\right\} \\
& \times \overline{\left\{\int_{\mathfrak{D}+u(s)} f(x) \overline{\varphi_{j, \lambda}(x+u(r))} d x\right\}} \\
& =\sum_{\lambda=0}^{(q N)^{j}-1} \sum_{r \in \mathbb{N}_{0}} \sum_{s \in \mathbb{N}_{0}}\left\{\int_{\mathbb{K}} F_{0}(x) \overline{\varphi_{j, \lambda}(x+u(r))} d x\right\} \\
& \times \overline{\left\{\int_{\mathbb{K}} F_{s}(x) \overline{\varphi_{j, \lambda}(x+u(r))} d x\right\}} \\
& =\sum_{\lambda=0}^{(q N)^{j}-1} \sum_{r \in \mathbb{N}_{0}} \sum_{s \in \mathbb{N}_{0}}\left\langle F_{0}, \varphi_{j, \lambda-\left(\mathfrak{p}^{-1} N\right)^{-j} u(r)}\right\rangle \overline{\left\langle F_{s}, \varphi_{j, \lambda-\left(\mathfrak{p}^{-1} N\right)^{-j} u(r)}\right\rangle} \\
& =\sum_{\lambda \in \Lambda} \sum_{s \in \mathbb{N}_{0}}\left\langle F_{0}, \varphi_{j, \lambda}\right\rangle \overline{\left\langle F_{s}, \varphi_{j, \lambda}\right\rangle} .
\end{aligned}
$$

Similarly, for each $1 \leq \ell \leq q N-1$, we have

$$
\sum_{\lambda=0}^{(q N)^{j}-1}\left|\left\langle f, \psi_{\ell, j, \lambda}^{\text {per }}\right\rangle\right|^{2}=\sum_{\lambda \in \Lambda} \sum_{s \in \mathbb{N}_{0}}\left\langle F_{0}, \psi_{\ell, j, \lambda}\right\rangle \overline{\left\langle F_{s}, \psi_{\ell, j, \lambda}\right\rangle} .
$$

By Lemma 3.2, we have

$$
\begin{aligned}
& \sum_{\lambda=0}^{(q N)^{j}-1}\left|\left\langle f, \varphi_{j, \lambda}^{\mathrm{per}}\right\rangle\right|^{2}+\sum_{\ell=1}^{q N-1} \sum_{\lambda=0}^{(q N)^{j}-1}\left|\left\langle f, \psi_{\ell, j, \lambda}^{\mathrm{per}}\right\rangle\right|^{2} \\
& \quad=\sum_{\lambda \in \Lambda} \sum_{s \in \mathbb{N}_{0}}\left\langle F_{0}, \varphi_{j, \lambda}\right\rangle \overline{\left\langle F_{s}, \varphi_{j, \lambda}\right\rangle}+\sum_{\ell=1}^{q N-1} \sum_{\lambda \in \Lambda} \sum_{s \in \mathbb{N}_{0}}\left\langle F_{0}, \psi_{\ell, j, \lambda}\right\rangle \overline{\left\langle F_{s}, \psi_{\ell, j, \lambda}\right\rangle}
\end{aligned}
$$




$$
=\sum_{\lambda \in \Lambda} \sum_{s \in \mathbb{N}_{0}}\left\langle F_{0}, \varphi_{j+1, \lambda}\right\rangle \overline{\left\langle F_{s}, \varphi_{j+1, \lambda}\right\rangle}=\sum_{\lambda=0}^{(q N)^{j}-1}\left|\left\langle f, \varphi_{j+1, \lambda}^{\text {per }}\right\rangle\right|^{2} .
$$

This completes the proof of Lemma 3.2.

Now we state and prove the main result of this section.

Theorem 3.1. Let $m_{0}(\xi)$ be the refinement mask of a refinable function $\varphi(x)$ and let $m_{\ell}(\xi), 1 \leq \ell \leq q N-1$, be the wavelet masks associated with the basic wavelets given by (2.13). Furthermore, let the wavelet system $\mathcal{W}(\Psi, \lambda)$ given by (2.7) form a normalized tight frame generated by the refinable function $\phi$. If $\left\{\varphi, \psi_{1}, \psi_{2}, \ldots, \psi_{q N-1}\right\} \subset L^{1}(\mathbb{K}) \cap L^{2}(\mathbb{K})$ and $\varphi, \psi_{1}, \psi_{2}, \ldots, \psi_{q N-1}$ have a common radial decreasing $L^{1}$-majorant, then the periodic wavelet system $\mathcal{W}^{\text {per }}(\Psi, \lambda)$ given by (3.1) generates a normalized tight frame for $L^{2}(\mathfrak{D})$.

Proof. For any periodic function $f \in \Omega(\mathfrak{D})$ and $\varepsilon>0$, we can choose $J>0$ by Lemma 3.1 such that for all $j>J$, we have

$$
(1-\varepsilon)\|f\|_{2}^{2} \leq \sum_{\lambda=0}^{(q N)^{j}-1}\left|\left\langle f, \varphi_{j, k}^{\text {per }}\right\rangle\right|^{2} \leq(1+\varepsilon)\|f\|_{2}^{2} .
$$

Also for any $j \in \mathbb{Z}$, Lemma 3.3 implies that

$$
\begin{aligned}
\sum_{\lambda=0}^{(q N)^{j}-1}\left|\left\langle f, \varphi_{j, \lambda}^{\mathrm{per}}\right\rangle\right|^{2}= & \sum_{\lambda=0}^{(q N) j-1-1}\left|\left\langle f, \varphi_{j-1, \lambda}^{\mathrm{per}}\right\rangle\right|^{2} \\
& +\sum_{\ell=1}^{q N-1} \sum_{\lambda=0}^{(q N)^{j-1}-1}\left|\left\langle f, \psi_{\ell, j-1, \lambda}^{\mathrm{per}}\right\rangle\right|^{2}
\end{aligned}
$$

Repeating the above argument on $\sum_{\lambda=0}^{(q N)^{j-1}-1}\left|\left\langle f, \varphi_{j-1, \lambda}^{\text {per }}\right\rangle\right|^{2}$, we obtain

$$
\sum_{\lambda=0}^{(q N)^{j}-1}\left|\left\langle f, \varphi_{j, \lambda}^{\mathrm{per}}\right\rangle\right|^{2}=\left|\left\langle f, \varphi^{\mathrm{per}}\right\rangle\right|^{2}+\sum_{\ell=1}^{q N-1} \sum_{r=0}^{j-1} \sum_{\lambda=0}^{(q N)^{r}-1}\left|\left\langle f, \psi_{\ell, r, \lambda}^{\mathrm{per}}\right\rangle\right|^{2} .
$$

Therefore, we have

$$
(1-\varepsilon)\|f\|_{2}^{2} \leq\left|\left\langle f, \varphi^{\mathrm{per}}\right\rangle\right|^{2}+\sum_{\ell=1}^{q N-1} \sum_{r=0}^{j-1} \sum_{\lambda=0}^{(q N)^{r}-1}\left|\left\langle f, \psi_{\ell, r, \lambda}^{\mathrm{per}}\right\rangle\right|^{2} \leq(1+\varepsilon)\|f\|_{2}^{2} .
$$

Letting $j \rightarrow \infty$, we obtain

$$
(1-\varepsilon)\|f\|_{2}^{2} \leq\left|\left\langle f, \varphi^{\mathrm{per}}\right\rangle\right|^{2}+\sum_{\ell=1}^{q N-1} \sum_{r \in \mathbb{N}_{0}} \sum_{\lambda=0}^{(q N)^{r}-1}\left|\left\langle f, \psi_{\ell, r, \lambda}^{\mathrm{per}}\right\rangle\right|^{2} \leq(1+\varepsilon)\|f\|_{2}^{2} .
$$


Since $\epsilon>0$ was arbitrary, it follows that

$$
\left|\left\langle f, \varphi^{\mathrm{per}}\right\rangle\right|^{2}+\sum_{\ell=1}^{q N-1} \sum_{r \in \mathbb{N}_{0}} \sum_{\lambda=0}^{(q N)^{r}-1}\left|\left\langle f, \psi_{\ell, r, \lambda}^{\mathrm{per}}\right\rangle\right|^{2}=\|f\|_{2}^{2} .
$$

This completes the proof of Theorem 3.1.

\section{REFERENCES}

[1] Ahmad, O., Sheikh, N. A., Ali, M. A., Nonuniform nonhomogeneous dual wavelet frames in Sobolev spaces in $L^{2}(\mathbb{K})$, Afrika Math. 31 (2020), 1145-1156.

[2] Ahmad, O., Sheikh, N. A., On Characterization of nonuniform tight wavelet frames on local fields, Anal. Theory Appl. 34 (2018), 135-146.

[3] Ahmad, O., Shah, F. A., Sheikh, N. A., Gabor frames on non-Archimedean fields, Int. J. Geom. Methods Mod. Phys. 15 (5) (2018), 1850079, 17 pp.

[4] Ahmad, O., Ahmad, N., Explicit construction of tight nonuniform framelet packets on local fields, Oper. Matrices (to appear).

[5] Ahmad, O., Ahmad, N., Construction of nonuniform wavelet frames on nonArchimedean fields, Math. Phy. Anal. Geom. (to appear).

[6] Benedetto, J. J., Benedetto, R. L., A wavelet theory for local fields and related groups, J. Geom. Anal. 14 (2004), 423-456.

[7] Christensen, O., An Introduction to Frames and Riesz Bases, Second Edition, Birkhäuser, Boston, 2016.

[8] Daubechies, I., Han, B., Ron, A., Shen, Z., Framelets: MRA-based constructions of wavelet frames, Appl. Comput. Harmon. Anal. 14 (2003), 1-46.

[9] Daubechies, I., Grossmann, A., Meyer, Y., Painless non-orthogonal expansions, J. Math. Phys. 27 (5) (1986), 1271-1283.

[10] Duffin, R. J., Shaeffer, A. C., A class of nonharmonic Fourier series, Trans. Amer. Math. Soc. 72 (1952), 341-366.

[11] Gabardo, J. P., Nashed, M., Nonuniform multiresolution analyses and spectral pairs, J. Funct. Anal. 158 (1998), 209-241.

[12] Gabardo, J. P., Yu, X., Wavelets associated with nonuniform multiresolution analysis and one-dimensional spectral pairs, J. Math. Anal. Appl. 323 (2006), 798-817.

[13] Jiang, H. K., Li, D. F., Jin, N., Multiresolution analysis on local fields, J. Math. Anal. Appl. 294 (2004), 523-532.

[14] Lebedeva, E. A., Prestin, J., Periodic wavelet frames and time-frequency localization, Appl. Comput. Harmon. Anal. 37 (2014), 347-359.

[15] Lu, D., Li, D., Construction of periodic wavelet frames with dilation matrix, Front. Math. China. 9 (2014), 111-134.

[16] Ramakrishnan, D., Valenza, R. J., Fourier Analysis on Number Fields, Graduate Texts in Mathematics 186, Springer-Verlag, New York, 1999.

[17] Ron, A., Shen, Z., Affine systems in $L^{2}\left(\mathbb{R}^{d}\right)$ : the analysis of the analysis operator, J. Funct. Anal. 148 (1997), 408-447.

[18] Shah, F. A., Ahmad, O., Jorgenson, P. E., Fractional wave packet frames in $L^{2}(\mathbb{R})$, J. Math. Phys. 59, 073509 (2018).

[19] Shah, F. A., Ahmad, O., Wave packet systems on local fields, J. Geom. Phys. 120 (2017), 5-18.

[20] Shah, F. A., Ahmad, O., Rahimi, A., Frames associated with shift invariant spaces on local fields, Filomat 32 (9)(2018), 3097-3110.

[21] Shah, F. A., Ahmad, O., Sheikh, N. A., Orthogonal Gabor systems on local fields, Filomat 31 (16) (2017), 5193-5201. 
[22] Shah, F. A., Ahmad, O., Sheikh, N. A., Some new inequalities for wavelet frames on local fields, Anal. Theory Appl. 33 (2) (2017), 134-148.

[23] Shah, F. A., Debnath, L., Tight wavelet frames on local fields, Analysis 33 (2013), 293-307.

[24] Taibleson, M. H., Fourier Analysis on Local Fields, Princeton University Press, Princeton, NJ, 1975.

[25] Zhang, Z., Periodic wavelet frames, Adv. Comput. Math. 22 (2005), 165-180.

[26] Zhang, Z., Saito, N., Constructions of periodic wavelet frames using extension principles, Appl. Comput. Harmon. Anal. 27 (2009), 12-23.

Owais Ahmad

Department of Mathematics

National Institute of Technology

Srinagar-190006

Jammu and Kashmir

India

e-mail: siawoahmad@gmail.com

Received September 4, 2020 